\title{
Search for new particles in the two-jet decay channel with the DO detector
}

V.M. Abazov, ${ }^{21}$ B. Abbott, ${ }^{55}$ A. Abdesselam, ${ }^{11}$ M. Abolins,${ }^{48}$ V. Abramov, ${ }^{24}$ B.S. Acharya,${ }^{17}$ D.L. Adams, ${ }^{53}$ M. Adams ${ }^{35}$ S.N. Ahmed, ${ }^{20}$ G.D. Alexeev, ${ }^{21}$ A. Alton, ${ }^{47}$ G.A. Alves, ${ }^{2}$ E.W. Anderson, ${ }^{40}$ Y. Arnoud, ${ }^{9}$ C. Avila, ${ }^{5}$ V.V. Babintsev, ${ }^{24}$ L. Babukhadia, ${ }^{52}$ T.C. Bacon, ${ }^{26}$ A. Baden, ${ }^{44}$ S. Baffioni, ${ }^{10}$ B. Baldin, ${ }^{34}$ P.W. Balm, ${ }^{19}$ S. Banerjee, ${ }^{17}$ E. Barberis, ${ }^{46}$ P. Baringer, ${ }^{41}$ J. Barreto, ${ }^{2}$ J.F. Bartlett, ${ }^{34}$ U. Bassler, ${ }^{12}$ D. Bauer, ${ }^{38}$ A. Bean, ${ }^{41}$ F. Beaudette, ${ }^{11}$ M. Begel, ${ }^{51}$ A. Belyaev, ${ }^{33}$ S.B. Beri, ${ }^{15}$ G. Bernardi, ${ }^{12}$ I. Bertram, ${ }^{25}$ A. Besson, ${ }^{9}$ R. Beuselinck, ${ }^{26}$ V.A. Bezzubov, ${ }^{24}$ P.C. Bhat, ${ }^{34}$ V. Bhatnagar, ${ }^{15}$ M. Bhattacharjee, ${ }^{52}$ G. Blazey, ${ }^{36}$ F. Blekman, ${ }^{19}$ S. Blessing, ${ }^{33}$ A. Boehnlein, ${ }^{34}$ N.I. Bojko, ${ }^{24}$ T.A. Bolton, ${ }^{42}$

F. Borcherding, ${ }^{34}$ K. Bos, ${ }^{19}$ T. Bose,${ }^{50}$ A. Brandt, ${ }^{57}$ G. Briskin, ${ }^{56}$ R. Brock, ${ }^{48}$ G. Brooijmans, ${ }^{34}$ A. Bross, ${ }^{34}$ D. Buchholz, ${ }^{37}$ M. Buehler, ${ }^{35}$ V. Buescher, ${ }^{14}$ V.S. Burtovoi, ${ }^{24}$ J.M. Butler, ${ }^{45}$ F. Canelli, ${ }^{51}$ W. Carvalho, ${ }^{3}$ D. Casey, ${ }^{48}$

H. Castilla-Valdez, ${ }^{18}$ D. Chakraborty, ${ }^{36}$ K.M. Chan, ${ }^{51}$ S.V. Chekulaev, ${ }^{24}$ D.K. Cho, ${ }^{51}$ S. Choi, ${ }^{32}$ S. Chopra, ${ }^{53}$ D. Claes, ${ }^{49}$ A.R. Clark, ${ }^{28}$ B. Connolly, ${ }^{33}$ W.E. Cooper, ${ }^{34}$ D. Coppage, ${ }^{41}$ S. Crépé-Renaudin, ${ }^{9}$ M.A.C. Cummings, ${ }^{36}$ D. Cutts, ${ }^{56}$

H. da Motta, ${ }^{2}$ G.A. Davis, ${ }^{51}$ K. De,${ }^{57}$ S.J. de Jong, ${ }^{20}$ M. Demarteau, ${ }^{34}$ R. Demina, ${ }^{51}$ P. Demine, ${ }^{13}$ D. Denisov, ${ }^{34}$ S.P. Denisov, ${ }^{24}$ S. Desai, ${ }^{52}$ H.T. Diehl, ${ }^{34}$ M. Diesburg, ${ }^{34}$ S. Doulas, ${ }^{46}$ L.V. Dudko, ${ }^{23}$ S. Duensing, ${ }^{20}$ L. Duflot, ${ }^{11}$ S.R. Dugad, ${ }^{17}$

A. Duperrin, ${ }^{10}$ A. Dyshkant, ${ }^{36}$ D. Edmunds, ${ }^{48}$ J. Ellison, ${ }^{32}$ J.T. Eltzroth, ${ }^{57}$ V.D. Elvira, ${ }^{34}$ R. Engelmann, ${ }^{52}$ S. Eno, ${ }^{44}$

G. Eppley, ${ }^{58}$ P. Ermolov, ${ }^{23}$ O.V. Eroshin, ${ }^{24}$ J. Estrada, ${ }^{51}$ H. Evans, ${ }^{50}$ V.N. Evdokimov, ${ }^{24}$ D. Fein, ${ }^{27}$ T. Ferbel, ${ }^{51}$ F. Filthaut, ${ }^{20}$ H.E. Fisk, ${ }^{34}$ F. Fleuret, ${ }^{12}$ M. Fortner, ${ }^{36}$ H. Fox, ${ }^{37}$ S. Fu, ${ }^{50}$ S. Fuess, ${ }^{34}$ E. Gallas, ${ }^{34}$ A.N. Galyaev, ${ }^{24}$ M. Gao, ${ }^{50}$

V. Gavrilov, ${ }^{22}$ R.J. Genik II, ${ }^{25}$ K. Genser, ${ }^{34}$ C.E. Gerber, ${ }^{35}$ Y. Gershtein, ${ }^{56}$ G. Ginther, ${ }^{51}$ B. Gómez, ${ }^{5}$ P.I. Goncharov, ${ }^{24}$ H. Gordon, ${ }^{53}$ K. Gounder, ${ }^{34}$ A. Goussiou, ${ }^{26}$ N. Graf, ${ }^{53}$ P.D. Grannis, ${ }^{52}$ J.A. Green, ${ }^{40}$ H. Greenlee, ${ }^{34}$ Z.D. Greenwood, ${ }^{43}$ S. Grinstein, ${ }^{1}$ L. Groer, ${ }^{50}$ S. Grünendahl, ${ }^{34}$ S.N. Gurzhiev, ${ }^{24}$ G. Gutierrez, ${ }^{34}$ P. Gutierrez, ${ }^{55}$ N.J. Hadley, ${ }^{44}$ H. Haggerty, ${ }^{34}$ S. Hagopian, ${ }^{33}$ V. Hagopian, ${ }^{33}$ R.E. Hall, ${ }^{30}$ C. Han, ${ }^{47}$ S. Hansen, ${ }^{34}$ J.M. Hauptman, ${ }^{40}$ C. Hebert, ${ }^{41}$ D. Hedin, ${ }^{36}$ J.M. Heinmiller, ${ }^{35}$ A.P. Heinson, ${ }^{32}$ U. Heintz, ${ }^{45}$ M.D. Hildreth, ${ }^{39}$ R. Hirosky, ${ }^{59}$ J.D. Hobbs, ${ }^{52}$ B. Hoeneisen, ${ }^{8}$ J. Huang, ${ }^{38}$ Y. Huang, ${ }^{47}$ I. Iashvili, ${ }^{32}$ R. Illingworth, ${ }^{26}$ A.S. Ito, ${ }^{34}$ M. Jaffré, ${ }^{11}$ S. Jain, ${ }^{17}$ R. Jesik, ${ }^{26}$ K. Johns, ${ }^{27}$ M. Johnson, ${ }^{34}$ A. Jonckheere, ${ }^{34}$ H. Jöstlein, ${ }^{34}$ A. Juste, ${ }^{34}$ W. Kahl,${ }^{42}$ S. Kahn, ${ }^{53}$ E. Kajfasz, ${ }^{10}$ A.M. Kalinin, ${ }^{21}$

D. Karmanov, ${ }^{23}$ D. Karmgard, ${ }^{39}$ R. Kehoe, ${ }^{48}$ S. Kesisoglou, ${ }^{56}$ A. Khanov, ${ }^{51}$ A. Kharchilava, ${ }^{39}$ B. Klima, ${ }^{34}$ J.M. Kohli, ${ }^{15}$

A.V. Kostritskiy, ${ }^{24}$ J. Kotcher, ${ }^{53}$ B. Kothari, ${ }^{50}$ A.V. Kozelov, ${ }^{24}$ E.A. Kozlovsky, ${ }^{24}$ J. Krane, ${ }^{40}$ M.R. Krishnaswamy, ${ }^{17}$

P. Krivkova, ${ }^{6}$ S. Krzywdzinski, ${ }^{34}$ M. Kubantsev, ${ }^{42}$ S. Kuleshov, ${ }^{22}$ Y. Kulik, ${ }^{34}$ S. Kunori, ${ }^{44}$ A. Kupco, ${ }^{7}$ V.E. Kuznetsov, ${ }^{32}$

G. Landsberg, ${ }^{56}$ W.M. Lee, ${ }^{33}$ A. Leflat, ${ }^{23}$ F. Lehner, ${ }^{34, *}$ C. Leonidopoulos, ${ }^{50}$ J. Li, ${ }^{57}$ Q.Z. Li, ${ }^{34}$ J.G.R. Lima, ${ }^{3}$

D. Lincoln, ${ }^{34}$ S.L. Linn, ${ }^{33}$ J. Linnemann, ${ }^{48}$ R. Lipton, ${ }^{34}$ A. Lucotte, ${ }^{9}$ L. Lueking, ${ }^{34}$ C. Lundstedt, ${ }^{49}$ C. Luo, ${ }^{38}$ A.K.A. Maciel, ${ }^{36}$ R.J. Madaras, ${ }^{28}$ V.L. Malyshev, ${ }^{21}$ V. Manankov, ${ }^{23}$ H.S. Mao, ${ }^{4}$ T. Marshall, ${ }^{38}$ M.I. Martin, ${ }^{36}$ S.E.K. Mattingly, ${ }^{56}$ A.A. Mayorov, ${ }^{24}$ R. McCarthy, ${ }^{52}$ T. McMahon, ${ }^{54}$ H.L. Melanson, ${ }^{34}$ A. Melnitchouk, ${ }^{56}$ M. Merkin, ${ }^{23}$ K.W. Merritt, ${ }^{34}$

C. Miao,${ }^{56}$ H. Miettinen, ${ }^{58}$ D. Mihalcea, ${ }^{36}$ N. Mokhov, ${ }^{34}$ N.K. Mondal, ${ }^{17}$ H.E. Montgomery, ${ }^{34}$ R.W. Moore, ${ }^{48}$

Y.D. Mutaf, ${ }^{52}$ E. Nagy, ${ }^{10}$ F. Nang, ${ }^{27}$ M. Narain, ${ }^{45}$ V.S. Narasimham, ${ }^{17}$ N.A. Naumann, ${ }^{20}$ H.A. Neal, ${ }^{47}$ J.P. Negret, ${ }^{5}$ A. Nomerotski, ${ }^{34}$ T. Nunnemann, ${ }^{34}$ D. O'Neil, ${ }^{48}$ V. Oguri, ${ }^{3}$ B. Olivier, ${ }^{12}$ N. Oshima, ${ }^{34}$ P. Padley, ${ }^{58}$ K. Papageorgiou, ${ }^{35}$ N. Parashar, ${ }^{43}$ R. Partridge, ${ }^{56}$ N. Parua, ${ }^{52}$ A. Patwa, ${ }^{52}$ O. Peters, ${ }^{19}$ P. Pétroff, ${ }^{11}$ R. Piegaia, ${ }^{1}$ B.G. Pope, ${ }^{48}$

H.B. Prosper, ${ }^{33}$ S. Protopopescu, ${ }^{53}$ M.B. Przybycien, ${ }^{37, \dagger}$ J. Qian, ${ }^{47}$ R. Raja, ${ }^{34}$ S. Rajagopalan, ${ }^{53}$ P.A. Rapidis, ${ }^{34}$ N.W. Reay, ${ }^{42}$ S. Reucroft, ${ }^{46}$ M. Ridel, ${ }^{11}$ M. Rijssenbeek, ${ }^{52}$ F. Rizatdinova, ${ }^{42}$ T. Rockwell, ${ }^{48}$ C. Royon, ${ }^{13}$ P. Rubinov, ${ }^{34}$ R. Ruchti, ${ }^{39}$ B.M. Sabirov, ${ }^{21}$ G. Sajot, ${ }^{9}$ A. Santoro, ${ }^{3}$ L. Sawyer, ${ }^{43}$ R.D. Schamberger, ${ }^{52}$ H. Schellman, ${ }^{37}$

A. Schwartzman, ${ }^{1}$ E. Shabalina, ${ }^{35}$ R.K. Shivpuri, ${ }^{16}$ D. Shpakov, ${ }^{46}$ M. Shupe, ${ }^{27}$ R.A. Sidwell, ${ }^{42}$ V. Simak, ${ }^{7}$

V. Sirotenko, ${ }^{34}$ P. Slattery, ${ }^{51}$ R.P. Smith, ${ }^{34}$ G.R. Snow, ${ }^{49}$ J. Snow, ${ }^{54}$ S. Snyder, ${ }^{53}$ J. Solomon, ${ }^{35}$ Y. Song, ${ }^{57}$ V. Sorín, ${ }^{1}$ M. Sosebee, ${ }^{57}$ N. Sotnikova, ${ }^{23}$ K. Soustruznik, ${ }^{6}$ M. Souza, ${ }^{2}$ N.R. Stanton, ${ }^{42}$ G. Steinbrück, ${ }^{50}$ D. Stoker, ${ }^{31}$ V. Stolin, ${ }^{22}$ A. Stone, ${ }^{43}$ D.A. Stoyanova, ${ }^{24}$ M.A. Strang, ${ }^{57}$ M. Strauss, ${ }^{55}$ M. Strovink, ${ }^{28}$ L. Stutte, ${ }^{34}$ A. Sznajder, ${ }^{3}$ M. Talby, ${ }^{10}$ W. Taylor, ${ }^{52}$ S. Tentindo-Repond, ${ }^{33}$ S.M. Tripathi, ${ }^{29}$ T.G. Trippe, ${ }^{28}$ A.S. Turcot, ${ }^{53}$ P.M. Tuts, ${ }^{50}$ R. Van Kooten, ${ }^{38}$ V. Vaniev, ${ }^{24}$ N. Varelas, ${ }^{35}$ F. Villeneuve-Seguier, ${ }^{10}$ A.A. Volkov, ${ }^{24}$ A.P. Vorobiev, ${ }^{24}$ H.D. Wahl, ${ }^{33}$ Z.-M. Wang, ${ }^{52}$ J. Warchol, ${ }^{39}$

G. Watts, ${ }^{60}$ M. Wayne, ${ }^{39}$ H. Weerts, ${ }^{48}$ A. White, ${ }^{57}$ D. Whiteson, ${ }^{28}$ D.A. Wijngaarden, ${ }^{20}$ S. Willis, ${ }^{36}$ S.J. Wimpenny, ${ }^{32}$ J. Womersley, ${ }^{34}$ D.R. Wood, ${ }^{46}$ Q. Xu, ${ }^{47}$ R. Yamada, ${ }^{34}$ P. Yamin, ${ }^{53}$ T. Yasuda, ${ }^{34}$ Y.A. Yatsunenko, ${ }^{21}$ K. Yip, ${ }^{53}$ J. Yu, ${ }^{57}$ M. Zanabria, ${ }^{5}$ X. Zhang, ${ }^{55}$ H. Zheng, ${ }^{39}$ B. Zhou, ${ }^{47}$ Z. Zhou, ${ }^{40}$ M. Zielinski, ${ }^{51}$ D. Zieminska, ${ }^{38}$ A. Zieminski, ${ }^{38}$ V. Zutshi, ${ }^{36}$ E.G. Zverev, ${ }^{23}$ and A. Zylberstejn ${ }^{13}$

\section{(DØ Collaboration)}

\author{
${ }^{1}$ Universidad de Buenos Aires, Buenos Aires, Argentina \\ ${ }^{2}$ LAFEX, Centro Brasileiro de Pesquisas Físicas, Rio de Janeiro, Brazil \\ ${ }^{3}$ Universidade do Estado do Rio de Janeiro, Rio de Janeiro, Brazil \\ ${ }^{4}$ Institute of High Energy Physics, Beijing, People's Republic of China \\ ${ }^{5}$ Universidad de los Andes, Bogotá, Colombia \\ ${ }^{6}$ Charles University, Center for Particle Physics, Prague, Czech Republic \\ ${ }^{7}$ Institute of Physics, Academy of Sciences, Center for Particle Physics, Prague, Czech Republic \\ ${ }^{8}$ Universidad San Francisco de Quito, Quito, Ecuador \\ ${ }^{9}$ Laboratoire de Physique Subatomique et de Cosmologie, IN2P3-CNRS, Universite de Grenoble 1, Grenoble, France
}


${ }^{10} C P P M$, IN2P3-CNRS, Université de la Méditerranée, Marseille, France

${ }^{11}$ Laboratoire de l'Accélérateur Linéaire, IN2P3-CNRS, Orsay, France

${ }^{12}$ LPNHE, Universités Paris VI and VII, IN2P3-CNRS, Paris, France

${ }^{13}$ DAPNIA/Service de Physique des Particules, CEA, Saclay, France

${ }^{14}$ Universität Mainz, Institut für Physik, Mainz, Germany

${ }^{15}$ Panjab University, Chandigarh, India

${ }^{16}$ Delhi University, Delhi, India

${ }^{17}$ Tata Institute of Fundamental Research, Mumbai, India

${ }^{18}$ CINVESTAV, Mexico City, Mexico

${ }^{19}$ FOM-Institute NIKHEF and University of Amsterdam/NIKHEF, Amsterdam, The Netherlands

${ }^{20}$ University of Nijmegen/NIKHEF, Nijmegen, The Netherlands

${ }^{21}$ Joint Institute for Nuclear Research, Dubna, Russia

${ }^{22}$ Institute for Theoretical and Experimental Physics, Moscow, Russia

${ }^{23}$ Moscow State University, Moscow, Russia

${ }^{24}$ Institute for High Energy Physics, Protvino, Russia

${ }^{25}$ Lancaster University, Lancaster, United Kingdom

${ }^{26}$ Imperial College, London, United Kingdom

${ }^{27}$ University of Arizona, Tucson, Arizona 85721, USA

${ }^{28}$ Lawrence Berkeley National Laboratory and University of California, Berkeley, California 94720, USA

${ }^{29}$ University of California, Davis, California 95616, USA

${ }^{30}$ California State University, Fresno, California 93740, USA

${ }^{31}$ University of California, Irvine, California 92697, USA

${ }^{32}$ University of California, Riverside, California 92521, USA

${ }^{33}$ Florida State University, Tallahassee, Florida 32306, USA

${ }^{34}$ Fermi National Accelerator Laboratory, Batavia, Illinois 60510, USA

${ }^{35}$ University of Illinois at Chicago, Chicago, Illinois 60607, USA

${ }^{36}$ Northern Illinois University, DeKalb, Illinois 60115, USA

${ }^{37}$ Northwestern University, Evanston, Illinois 60208, USA

${ }^{38}$ Indiana University, Bloomington, Indiana 47405, USA

${ }^{39}$ University of Notre Dame, Notre Dame, Indiana 46556, USA

${ }^{40}$ Iowa State University, Ames, Iowa 50011, USA

${ }^{41}$ University of Kansas, Lawrence, Kansas 66045, USA

${ }^{42}$ Kansas State University, Manhattan, Kansas 66506, USA

${ }^{43}$ Louisiana Tech University, Ruston, Louisiana 71272, USA

${ }^{44}$ University of Maryland, College Park, Maryland 20742, USA

${ }^{45}$ Boston University, Boston, Massachusetts 02215, USA

${ }^{46}$ Northeastern University, Boston, Massachusetts 02115, USA

${ }^{47}$ University of Michigan, Ann Arbor, Michigan 48109, USA

${ }^{48}$ Michigan State University, East Lansing, Michigan 48824, USA

${ }^{49}$ University of Nebraska, Lincoln, Nebraska 68588, USA

${ }^{50}$ Columbia University, New York, New York 10027, USA

${ }^{51}$ University of Rochester, Rochester, New York 14627, USA

${ }^{52}$ State University of New York, Stony Brook, New York 11794, USA

${ }^{53}$ Brookhaven National Laboratory, Upton, New York 11973, USA

${ }^{54}$ Langston University, Langston, Oklahoma 73050, USA

${ }^{55}$ University of Oklahoma, Norman, Oklahoma 73019, USA

${ }^{56}$ Brown University, Providence, Rhode Island 02912, USA

${ }^{57}$ University of Texas, Arlington, Texas 76019, USA

${ }^{58}$ Rice University, Houston, Texas 77005, USA

${ }^{59}$ University of Virginia, Charlottesville, Virginia 22901, USA

${ }^{60}$ University of Washington, Seattle, Washington 98195, USA

(Received 13 August 2003; published 2 June 2004) 
We present the results of a search for the production of new particles decaying into two jets in $\bar{p} p$ collisions at $\sqrt{s}=1.8 \mathrm{TeV}$, using the $\mathrm{D} \emptyset 1992-1995$ data set corresponding to $109 \mathrm{pb}^{-1}$. We exclude at the $95 \%$ confidence level the production of excited quarks $\left(q^{*}\right)$ with masses below $775 \mathrm{GeV} / c^{2}$, the most restrictive limit to date. We also exclude standard-model-like $W^{\prime}\left(Z^{\prime}\right)$ bosons with masses between 300 and $800 \mathrm{GeV} /$ $c^{2}\left(400\right.$ and $\left.640 \mathrm{GeV} / \mathrm{c}^{2}\right)$. A $W^{\prime}$ boson with mass $<786 \mathrm{GeV} / c^{2}$ has been excluded by previous measurements, and our lower limit is therefore the most stringent to date.

DOI: 10.1103/PhysRevD.69.111101

PACS number(s): 13.85.Rm, 14.70.Pw, 14.80.-j

The direct production of hadronic jets is the dominant contribution to high transverse momentum $\left(p_{T}\right)$ processes in antiproton-proton $(\bar{p} p)$ collisions. There are many extensions of the standard model that predict the existence of new massive objects (e.g., excited quarks [1], $W^{\prime}$ and $Z^{\prime}$ bosons) that couple to quarks and/or gluons and may be observed as resonant structures in the two-jet mass spectrum. The previous observation of $W$ and $Z$ bosons decaying into two jets in the UA2 experiment [2] proved the feasibility of doing dijet mass spectroscopy at $\bar{p} p$ colliders. Subsequently, the UA2 [3] and CDF [4] experiments searched for new resonances in the dijet mass spectrum and set limits on their production within the context of different theoretical models. This Rapid Communication reports on a search for such resonances in the two-jet mass spectrum $[5,6]$ using the data collected at a center-of-mass energy of $1.8 \mathrm{TeV}$ with the D $\emptyset$ detector in 1992-1995, corresponding to an integrated luminosity of $109 \mathrm{pb}^{-1}$.

Jet detection in the $\mathrm{D} \emptyset$ detector [7] primarily utilizes the uranium/liquid-argon calorimeters that cover the pseudorapidity region $|\eta| \lesssim 4$, where $\eta=-\ln [\tan (\theta / 2)]$ and $\theta$ is the polar angle with respect to the proton beam. Jets are reconstructed using an iterative jet cone algorithm with a cone radius of $\mathcal{R}=0.7$ in $\eta$ - $\phi$ space [5], where $\phi$ is the azimuthal angle. Background jets from isolated noisy calorimeter cells and accelerator losses are minimized via jet-quality criteria [5]. The transverse energy of each jet is then corrected [8] for offsets due to the underlying event, noise, multiple interactions and pileup, the fraction of particle energy showering outside of the jet cone, and calorimeter energy response to incident hadrons.

For each event that passes the quality criteria, the inclusive dijet mass can be calculated, assuming that the particles within the jets are massless, using the relationship $M^{2}$ $=2 E_{T}^{1} E_{T}^{2}[\cosh (\Delta \eta)-\cos (\Delta \phi)]$, where $E_{T}^{1}$ and $E_{T}^{2}$ are the transverse energies of the two highest- $E_{T}$ jets. The pseudorapidities of the two leading jets are selected to be $\left|\eta_{1,2}\right|$ $<1.0$ and $\Delta \eta=\left|\eta_{1}-\eta_{2}\right|<1.6$ in order to maximize the range of dijet mass at which the trigger is efficient.

A single trigger was used to collect the 1992-1993 data, with an $E_{T}$ threshold of $115 \mathrm{GeV}$, for an integrated luminosity of $14.1 \mathrm{pb}^{-1}$. During 1994-1995, the data were collected using four triggers, with uncorrected $E_{T}$ thresholds of 30, 50, 85 and $115 \mathrm{GeV}$, for integrated luminosities of $0.36,4.8$, 56.5 , and $94.9 \mathrm{pb}^{-1}$, respectively. After the jet-energy cor-

\footnotetext{
*Visitor from University of Zurich, Zurich, Switzerland.

${ }^{\dagger}$ Visitor from Institute of Nuclear Physics, Krakow, Poland.
}

rections, these trigger samples are used to measure the dijet mass spectrum above mass thresholds of 180, 250, 320, and $470 \mathrm{GeV} / c^{2}$, respectively, where each of the triggers is $>97 \%$ efficient. The resulting dijet mass spectrum is shown in Fig. 1. The widths of the mass bins are chosen such that all events in any bin are recorded using a single trigger, there were $>10$ events per bin, and the bin width is approximately equal to the mass resolution.

The uncertainty in the dijet mass spectrum from the uncertainty in luminosity is $5.8 \%$, and the uncertainty from the jet-quality and vertex criteria is $1 \%$. The uncertainties due to the jet energy scale [8] are 7\% (30\%) for the lowest (highest) mass bin, and are correlated. The uncertainty in energy scale has three components: the uncorrelated, fully correlated, and partially-correlated uncertainties. A correlation matrix is calculated for the partially correlated uncertainties using the method described in Ref. [5]. The uncertainties in the mass spectra due to the jet energy resolution are (0.5-3.0)\% over the mass range under consideration.

Multijet background was simulated using the next-toleading order (NLO) program JETRAD [9], with the CTEQ6M [10] PDFs, and renormalization scale $(\mu)$ of $0.5 E_{T}^{\max }$, where the $E_{T}^{\max }$ is the $E_{T}$ of the highest- $E_{T}$ parton. Partons within $1.3 \mathcal{R}$ of one another are clustered into a single jet if they are within $\mathcal{R}=0.7$ of their $E_{T}$-weighted $\eta, \phi$ centroid [5]. The two highest- $E_{T}$ jets are used to calculate the dijet mass, which is then smeared using the measured mass resolutions. The resulting distribution is shown in Fig. 2.

A comparison between the background prediction and the data is given in Fig. 3 (only uncorrelated uncertainties are shown). The $\chi^{2}$ of the comparison is 25.0 for 25 degrees of

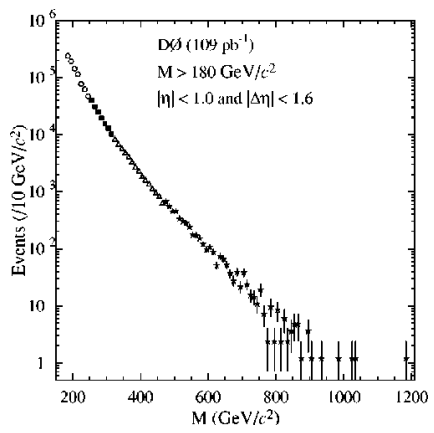

FIG. 1. The inclusive dijet mass spectrum. The events from each trigger have been corrected by the trigger's luminosity and event efficiency. The data were collected using triggers with uncorrected $E_{T}$ thresholds of 30 (open circles), 50 (closed squares), 85 (open triangles), and $115 \mathrm{GeV}$ (closed stars). The error bars represent statistical uncertainties. 


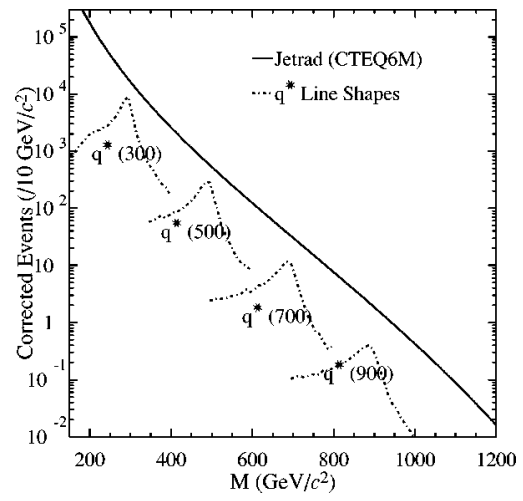

FIG. 2. The JETRAD (solid line) simulation of the inclusive dijet mass spectrum. The dashed-dotted lines show PYTHIA simulations of the excited quark line shapes for $M_{q^{*}}=300,500,700$, and $900 \mathrm{GeV} / c^{2}$.

freedom. This fit shows no obvious evidence for the existence of new particles.

We consider three models for a possible signal in the dijet mass spectrum. The first model contains a mass-degenerate family of excited quarks [1] that decay to a quark and a gluon $\left(q^{*} \rightarrow q g\right)$. We assume that the coupling parameters of the excited quarks equal unity $\left(f=f^{\prime}=f_{s}=1\right)$ and that the compositeness scale equals the mass of the excited quark $\left(\Lambda *=M_{q} *\right.$ ). The second and third models contain additional $W$ and $Z$ bosons, respectively, with standard-model-like couplings, where all possible quark decays are allowed $\left(W^{\prime}\right.$ $\rightarrow q \bar{q}^{\prime}, Z^{\prime} \rightarrow q \bar{q}$, with $W^{\prime} \rightarrow t \bar{b}$, and $Z^{\prime} \rightarrow t \bar{t}$ allowed when kinematically possible). The leading-order $W^{\prime}$ and $Z^{\prime}$ boson production cross sections are corrected by NLO " $K$ factors" [11] of approximately 1.3 , to account for higherorder effects. All models were generated using PYTHIA 6.2 [12], with the CTEQ6 parton distribution functions (PDFs) $[10]$.

For each of the models, a Monte Carlo mass spectrum was generated at $25 \mathrm{GeV} / c^{2}$ intervals from a mass of $150 \mathrm{GeV} / c^{2}$ to $1 \mathrm{TeV} / c^{2}$. Jets are reconstructed at the particle level using the same iterative jet cone algorithm that is

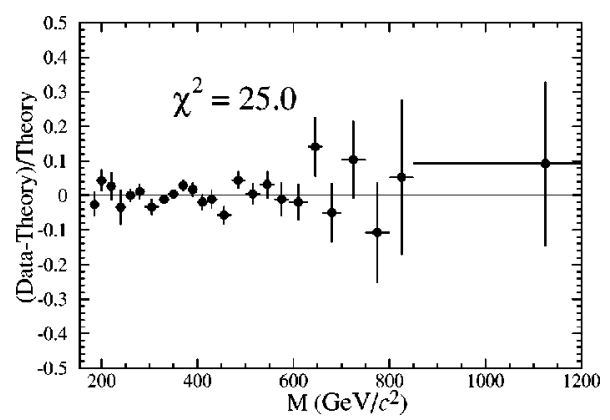

FIG. 3. The difference between data and the smeared JETRAD NLO QCD prediction normalized to the theoretical prediction ((Data-Theory)/Theory) using the CTEQ6M PDFs and a single renormalization scale $\mu=0.5 E_{T}^{\max }$. The vertical error bars represent the sum of the uncorrelated uncertainties added in quadrature, while the horizontal error bands represent the widths of the mass bins. The highest mass bin extends to $1400 \mathrm{GeV} / \mathrm{c}^{2}$.

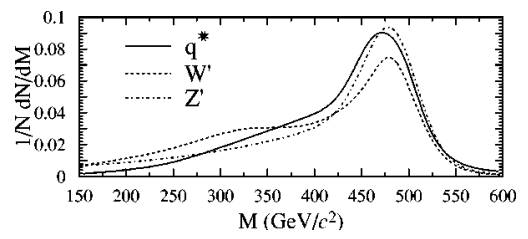

FIG. 4. The line shapes of a $500 \mathrm{GeV} / c^{2} q^{*}, W^{\prime}$ and $Z^{\prime}$ bosons, smoothed and normalized to unit area.

applied to the data. The resulting energies are then smeared with the measured jet resolutions. Each of the mass spectra contains 50000 events. Examples of the spectra generated for a resonant mass of $500 \mathrm{GeV} / \mathrm{c}^{2}$ are shown in Fig. 4.

The data were analyzed using a Bayesian technique, with a flat prior for the signal (see Ref. [13]). The predicted number of events per bin is given by $\mu_{i}=\left(\sigma_{Q C D_{i}} C_{Q C D_{i}}\right.$ $\left.+N_{X_{i}} \sigma_{X} C_{X_{i}}\right) \mathcal{L}_{i} \epsilon_{i}$ where $\sigma_{Q C D_{i}}$ is the predicted QCD two-jet cross section for mass bin $i ; N_{X_{i}}$ is the fraction of signal events in the bin $\left(\Sigma N_{X_{i}}=1\right) ; \sigma_{X}$ is the cross section for the signal; $\mathcal{L}_{i}$ is the integrated luminosity; $\boldsymbol{\epsilon}_{i}$ corresponds to the product of the efficiencies of the jet-quality criteria, the vertex selection efficiencies, and the trigger efficiencies per bin; and $C_{Q C D_{i}}$ and $C_{X_{i}}$ are the jet energy and resolution corrections on the background and signal, respectively. Assuming $N_{i}$ follows Poisson statistics, the probability that $N_{i}$ events are observed in a given mass bin is then given by $P\left(N_{i} \mid \sigma_{Q C D_{i}}, \sigma_{X}, N_{X_{i}}, \mathcal{L}, \epsilon_{i}, C_{X_{i}}, C_{Q C D_{i}}, I\right)=e^{-\mu_{i}} \mu_{i}^{N_{i} / N_{i} !}$,

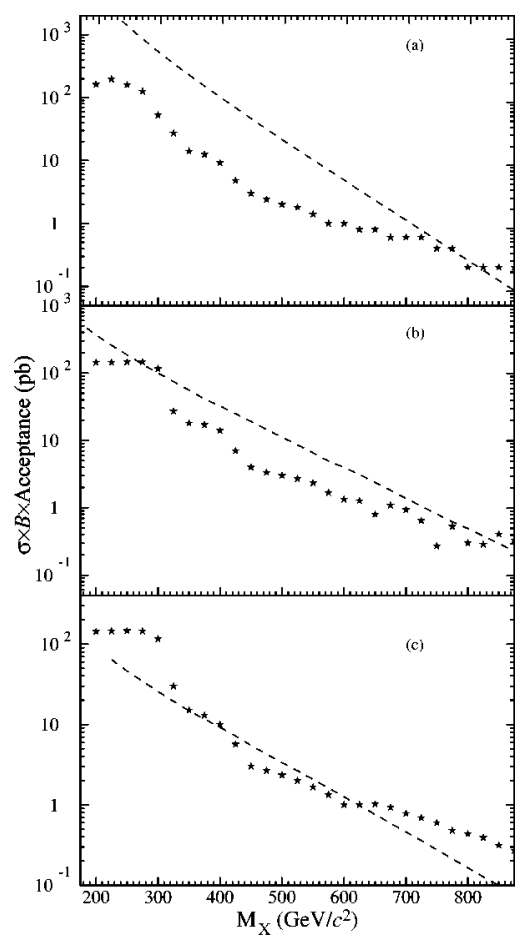

FIG. 5. The 95\% C.L. on the production cross section multiplied by $B(X \rightarrow$ dijet $)$ and acceptance, using the CTEQ6M PDFs for: (a) an excited quark $q^{*}$ (stars), compared with the predicted cross section (dashed line); $M_{q^{*}}<775 \mathrm{GeV} / c^{2}$ is excluded; (b) similarly, for a $W^{\prime}$ boson (stars), $300<M_{W^{\prime}}<800 \mathrm{GeV} / c^{2}$ is excluded; and (c) for a $Z^{\prime}$ boson (stars), $400<M_{Z^{\prime}}<640 \mathrm{GeV} / c^{2}$ is excluded. 
where $I$ reflects all other assumed information, such as the standard model parameters. The probability of observing the set $N_{i}$ that makes up the mass spectrum is then given by the product of these probabilities. To calculate the probability distribution for $\sigma_{X}$, Bayes' theorem is applied with the following assumptions about the prior probability distributions: $\sigma_{X}$ has a uniform prior; $\sigma_{Q C D_{i}}, \epsilon_{i}, C_{Q C D_{i}}, C_{X_{i}}$ and $\mathcal{L}_{i}$ all have Gaussian priors with widths given by their uncertainties; and $N_{i}$ has a Poisson prior. All uncertainties represented by Gaussian priors have means that are many standard deviations from any physical boundaries.

The $95 \%$ confidence level (C.L.) limits on the production cross sections for the three signal models are extracted. In order to convert these production cross section upper limits into lower limits on the mass of a new particle resonance, we compare (see Fig. 5) our measured 95\% C.L. limits (stars) with the expected cross section multiplied by the branching fraction $(B)$ and acceptance for particles decaying to dijets (dashed curve). Branching fractions to all possible quark and gluon states are included in the acceptance. The acceptances for excited quarks ( $W^{\prime}$ and $Z^{\prime}$ bosons) range from $20 \%$ at $200 \mathrm{GeV} / c^{2}$ to $60 \%(50 \%)$ for masses above $700 \mathrm{GeV} / c^{2}$. We exclude excited quarks with $M_{q}<775 \mathrm{GeV} / c^{2}$. This is the most restrictive limit on excited quark production to date. A $W^{\prime}$ boson is ruled out in the mass range $300<M_{W^{\prime}}$ $<800 \mathrm{GeV} / c^{2}$. Previous measurements [14,15] have excluded a $W^{\prime}$ boson with mass below $786 \mathrm{GeV} / c^{2}$; our new measurement therefore sets a more stringent lower limit on a $W^{\prime}$ boson mass of $800 \mathrm{GeV} / c^{2}$. A $Z^{\prime}$ boson with mass between 400 and $640 \mathrm{GeV} / c^{2}$ is also excluded (a previous measurement by CDF excludes a $Z^{\prime}$ boson below $690 \mathrm{GeV} /$ $\left.c^{2} \quad[16]\right)$.

We thank the staffs at Fermilab and collaborating institutions, and acknowledge support from the Department of Energy and National Science Foundation (USA), Commissariat à L'Energie Atomique and CNRS/Institut National de Physique Nucléaire et de Physique des Particules (France), Ministry for Science and Technology and Ministry for Atomic Energy (Russia), CAPES, CNPq and FAPERJ (Brazil), Departments of Atomic Energy and Science and Education (India), Colciencias (Colombia), CONACyT (Mexico), Ministry of Education and KOSEF (Korea), CONICET and UBACyT (Argentina), The Foundation for Fundamental Research on Matter (The Netherlands), PPARC (United Kingdom), Ministry of Education (Czech Republic), A.P. Sloan Foundation, and the Research Corporation.
[1] U. Baur, I. Hinchliffe, and D. Zeppenfeld, Int. J. Mod. Phys. A 2, 1285 (1987); U. Baur, M. Spira, and P.M. Zerwas, Phys. Rev. D 42, 815 (1990).

[2] UA2 Collaboration, J. Alitti et al., Z. Phys. C 49, 17 (1991).

[3] UA2 Collaboration, J. Alitti et al., Nucl. Phys. B400, 3 (1993).

[4] CDF Collaboration, F. Abe et al., Phys. Rev. D 55, 5263 (1997)

[5] DØ Collaboration, B. Abbott et al., Phys. Rev. D 64, 032003 (2001).

[6] DØ Collaboration, B. Abbott et al., Phys. Rev. Lett. 82, 2457 (1999).

[7] D $\emptyset$ Collaboration, S. Abachi et al., Nucl. Instrum. Methods Phys. Res. A 338, 185 (1994).

[8] DØ Collaboration, B. Abbott et al., Nucl. Instrum. Methods Phys. Res. A 424, 352 (1999).
[9] W.T. Giele, E.W.N. Glover, and D.A. Kosower, Phys. Rev. Lett. 73, 2019 (1994).

[10] J. Pumplin et al., J. High Energy Phys. 07, 12 (2002).

[11] R. Hamberg, W.L. van Neerven, and T. Matsuura, Nucl. Phys. B359, 343 (1991).

[12] T. Sjöstrand et al., Comput. Phys. Commun. 135, 238 (2001).

[13] I. Bertram et al., "A Recipe for the Construction of Confidence Limits," FERMILAB-TM-2104 (2000).

[14] CDF Collaboration, T. Affolder et al., Phys. Rev. Lett. 87, 231803 (2001).

[15] D $\emptyset$ Collaboration, S. Abachi et al., Phys. Rev. Lett. 76, 3271 (1996).

[16] CDF Collaboration, F. Abe et al., Phys. Rev. Lett. 79, 2192 (1997). 